\title{
Study on Physical Simulation Test for Pipeline Vulnerability in Case of Landslide
}

\author{
Dongjie Tan', Luyao Bai', Yongjian Yang², Ning Shi' ${ }^{1}$, Chai Li ${ }^{3}$ \\ ${ }^{1}$ National Engineering Laboratory of Oil \& Gas Pipeline Transportation Safety, Petro China Pipeline R \& D Center, Langfang, \\ China \\ ${ }^{2}$ Xi'an Oil \& Gas Transmission Sub-Company, Petro China Pipeline Company, Xi'an, China \\ ${ }^{3}$ Institution of Geological Surveying and Mapping of Hebei Province, Langfang, China \\ Email: tdj@petrochina.com.cn, bailuyao@petrochina.com.cn
}

How to cite this paper: Tan, D.J., Bai, L.Y., Yang, Y.J., Shi, N. and Li, C. (2018) Study on Physical Simulation Test for Pipeline Vulnerability in Case of Landslide. Journal of Geoscience and Environment Protection, 6, 70-79.

https://doi.org/10.4236/gep.2018.65006

Received: March 30, 2018

Accepted: May 20, 2018

Published: May 23, 2018

\begin{abstract}
Landslide is one of the most harmful geological disasters for long-distance oil and gas pipelines, and evaluating pipeline vulnerability in case of landslide consequently plays an important role in improving the landslide risk assessment level. To ensure creditability, applicability and operability of pipeline vulnerability evaluation results, a physical simulation test based on the similarity principle was carried out between landslide and pipeline to verify the deformation behavior, stress and strain distribution status of pipeline in case of landslide and to acquire the empirical formula of pipeline vulnerability and distribution of thrust applied on the pipeline by landslide.
\end{abstract}

\section{Keywords}

Oil and Gas Pipeline, Landslide, Physical Simulation, Vulnerability

\section{Introduction}

Geological disasters severely endanger safe operation of the long-distance oil and gas pipeline, in which, landslide is the most severe [1]. In March 1987, a giant landslide caused by earthquake led to a $40 \mathrm{~km}$ long fracture zone in the pipeline traversing Ecuador, resulting in shutdown for two weeks and economic losses about USD 700 million. In 1997 and 1999, two accidents occurred to pipelines with a diameter of 26 inches in Washington State of America due to landslide. Given enormous investments for landslide control, it is impossible and unnecessary to manage all disasters. Landslide collapse risk assessment for oil and gas pipelines is required and its vulnerability evaluation is the key link.

During study on pipeline disaster vulnerability, a simplified pipeline-geological 
body model is generally established, to complete calculation and analysis through analytical method or finite element method in combination with monitoring data. For example, Italy SNAM studies the pipeline affected by landslide featured with slow movement through the numerical simulation method based on living examples and compares it with the monitoring results for verification [2]. Chinese scholars also complete force analysis for pipeline affected by landslide. For instance, Chen Liqiong, et al. analyzed the stress of gas pipelines running through landslide areas longitudinally and transversally by using CAESAR II and ANSYS based on the finite element method. Then, the effects of the displacement and soil properties of landslides and the OD, wall thickness, internal pressure and material of pipes on the stress and strain of pipelines were analyzed [3]. Liu Bing, et al. conducted finite element analyzed (FEA) for a total of 594 cases covering a wide range of materials, ratios of pipe diameter to wall thickness, D/t and various internal pressures and developed parametric equations for strain limits of pipelines [4]. Han Bing, et al. analyzed the distribution of pipe strain caused by landslide through which the pipeline passes based on the general finite element program ABAQUS [5]. Hao Jianbin, et al. analyzed the interaction between landside and pipeline and deduced a formula for calculating landslide thrust force to transverse pipelines by using a limit equilibrium method [6]. Lian Zhangfu et al. successively simulate the force conditions of pipeline under different influence factors with the AUAQUS finite element software, providing the basis for safe operation of pipeline in case of landslide [7].

The numerical simulation has certain limitations due to many influence factors for pipeline landslide and its complex formation process while the physical simulation enables the optimization to mechanical model and improvement for simulation accuracy. As indicated by theoretical study and practice, the interactive relationship between geo-hazards body and oil and gas pipeline is closely related to the pipeline status. Therefore, the physical simulation method is adopted to simulate and verify the deformation behavior, its stress and strain distribution status of oil and gas pipeline under effects of geo-hazards body, to acquire action characteristics and expression of pipeline and calculate the maximum additional stress through the expression.

\section{Physical Model for Pipeline Vulnerability in Case of Landslide}

Based on the similarity principle, a landslide-pipeline generalized model is established and a physical simulation test is completed for the pipeline suffering landslide, with the geometric dimension scale of 1:50. The design landslide area is $45 \mathrm{~m}$ long, $30 \mathrm{~m}$ wide and $10 \mathrm{~m}$ thick and both ends of pipeline are only constrained by soil mass. The process from stable landslide to pipeline damage can be simulated through rotating the model and adjusting its gradient. It is used as the standard test model and its various influence factors may be changed, to analyze force and deformation of pipeline in case of landslide. See Figure 1 and Figure 2 for the model. 
The copper pipe, with an outer diameter of $1 \mathrm{~cm}$, an inner diameter of $8 \mathrm{~mm}$ and a length of $2 \mathrm{~m}$, is used as the pipeline. While the pipeline traverses the landslide, its right side is pasted with 19 large strain gages in axial direction and 19 small strain gages transversally close to the large strain gages; 5 large strain gages and 5 small strain gages are respectively pasted on the reverse side of pipeline in the axial direction and the transverse direction. For the 10 strain gages, one large and small strain gage are located in the center, two pairs of large and small strain gages are pasted at the edge of landslide body and two pairs of large and small strain gages are pasted outside of the landslide body. All of them are pasted symmetrically. While the pipeline passes through the landslide longitudinally, the pipeline may be affected by the torsional force, resulting in different pasting mode in the upper part compared to that of pipeline transversely crossing landslide, as shown in Figure 3.

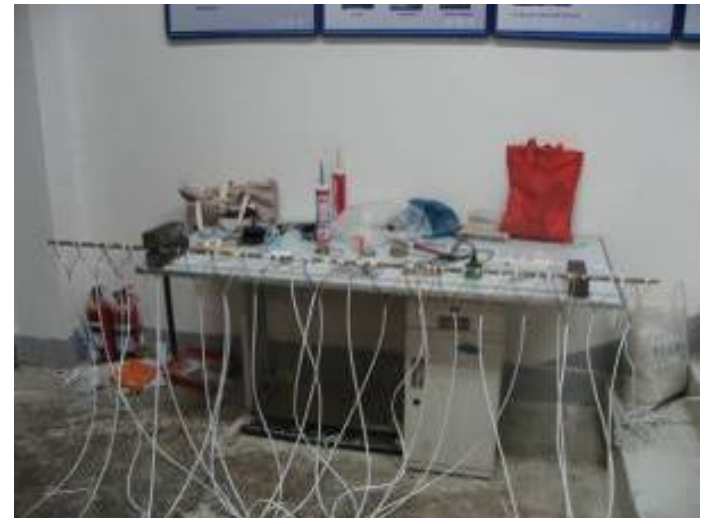

Figure 1. Pipeline model.

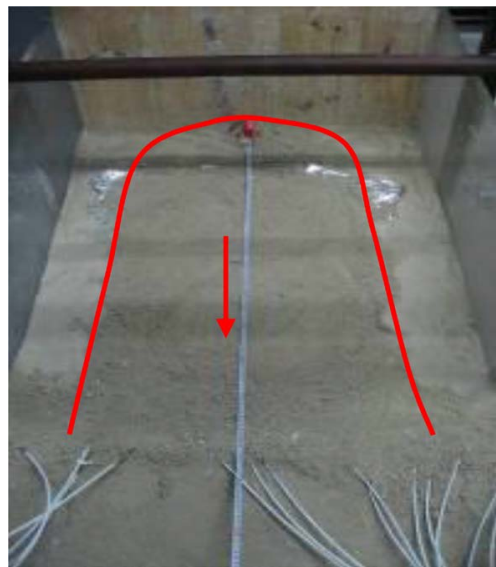

Figure 2. Landslide model.

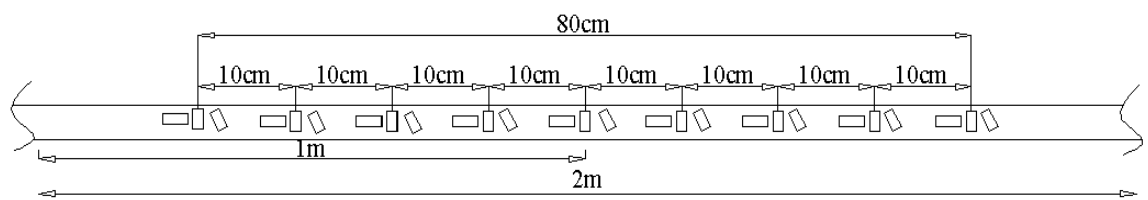

Figure 3. Pasting diagram of strain gages for pipeline crossing landslide longitudnally. 


\section{Test Scheme}

Pipeline crossing landslide areas transversely, longitudinally and diagonally are considered. The design test for pipeline transversely crossing landslide area includes 6 groups of test models, mainly studying thickness, length, width of landslide body, buried depth of pipeline, constraint mode and parameter indexes of sliding surface; the design test for pipeline longitudinally crossing landslide area includes 3 groups of test models, mainly studying thickness of landslide body, parameter indexes and form of sliding surface and included angle between pipeline and landslide body; crossing variety and complexity of pipeline diagonally crossing landslide are richer than that of two aforementioned types and they shall be significantly simplified, for which, only variation of included angle between pipeline and sliding direction of landslide, included in the model, is considered.

Each group of models is to change a certain influence factor on the basis of the standard landslide model. During the test, necessary stress and deformation monitoring points are set in the pipeline and deformation monitoring points (in surface and deep parts) are also set in the entire landslide model. The pipeline transversely crossing landslide is taken as an example and basic information of each model is as follows.

1) Varying with thickness

Thicknesses of model are $10 \mathrm{~cm}, 15 \mathrm{~cm}, 18 \mathrm{~cm}$ and $20 \mathrm{~cm}$ respectively. The considered length of landslide area is $90 \mathrm{~cm}$, the width is $60 \mathrm{~cm}$ and the buried depth of pipeline is $4 \mathrm{~cm}$ (from top surface to slope surface); substances of landslide (i.e. spring constraint) are set on both ends and the internal friction angle $(\Phi)$ of sliding surface is $22^{\circ}$. Based on test results, the potential influence depth is identified for infill test.

2) Varying with width

In the model, the width is set as $40 \mathrm{~cm}, 50 \mathrm{~cm}, 60 \mathrm{~cm}$ and $70 \mathrm{~cm}$, respectively; the length of landslide area is set as $90 \mathrm{~cm}$, the thickness as $20 \mathrm{~cm}$, and the buried depth as $4 \mathrm{~cm}$ (from top surface to slope surface); substances of landslide (i.e. spring constraint) are set on both ends and the internal friction angle $(\Phi)$ of sliding surface is $22^{\circ}$.

3) Varying with length

In the model, the distances from pipeline to trailing edge of landslide are 50 $\mathrm{cm}, 60 \mathrm{~cm}, 70 \mathrm{~cm}, 80 \mathrm{~cm}$ and $90 \mathrm{~cm}$ respectively. The considered width of landslide area is $60 \mathrm{~cm}$, the thickness is $20 \mathrm{~cm}$ and the buried depth of pipeline is $4 \mathrm{~cm}$ (from top surface to slope surface); substances of landslide (i.e. spring constraint) are set on both ends and the internal friction angle $(\Phi)$ of sliding surface is $22^{\circ}$.

4) Variation of buried depth of pipeline

In the model, the distances from top surface of pipeline to slope surface are 2 $\mathrm{cm}, 4 \mathrm{~cm}, 6 \mathrm{~cm}, 8 \mathrm{~cm}$ and $10 \mathrm{~cm}$ respectively. The considered length of landslide area is $90 \mathrm{~cm}$, the width is $60 \mathrm{~cm}$ and the thickness is $20 \mathrm{~cm}$; substances of 
landslide (i.e. spring constraint) are set on both ends and the internal friction angle $(\Phi)$ of sliding surface is $22^{\circ}$.

5) Variation of pipeline constraint mode

Given influence from pipeline constraint mode, pipeline forces, under conditions with one end fixed, both ends fixed and both ends movable, are focused for consideration.

6) Varying with parameters

The considered internal friction angles $(\varphi)$ include $15^{\circ}, 22^{\circ}$ and $26^{\circ}$. The considered length of landslide area is $90 \mathrm{~cm}$, the width is $60 \mathrm{~cm}$, the thickness is 20 $\mathrm{cm}$ and the buried depth of pipeline is $4 \mathrm{~cm}$ (from top surface to slope surface); substances of landslide (i.e. spring constraint) are set on both ends.

\section{Result Analysis}

\subsection{Test Process and Phenomenon Analysis}

In each model, a certain influence factor (vulnerability evaluation index) of geo-hazards body to additional stress of pipeline is changed in each model, and stress and deformation of pipeline are measured when the landslide with different inclination angles is under the critical state, acquiring the expression of force applied by the landslide to the pipeline in critical state. The model with a landslide $90 \mathrm{~cm}$ long, $60 \mathrm{~cm}$ wide, $10 \mathrm{~cm}$ thick, a pipeline buried depth of $4 \mathrm{~cm}$ and internal friction angle of $22^{\circ}$ involved in the mode is taken as an example, to describe test process and analyze its results.

During the test, the inclination angle of sliding surface at $10^{\circ}, 15^{\circ}, 20^{\circ}, 25^{\circ}$, $27^{\circ}, 28.5^{\circ}, 30^{\circ}, 31^{\circ}$ and $31.5^{\circ}$ shall be kept for 5 minutes and the parameters concerned shall be measured. About $1.5 \mathrm{~mm}$ crack appears at the trailing edge when being lifted to $25^{\circ}$; about $4 \mathrm{~mm}$ surface deformation occurs at $27^{\circ} ; 5$ - 6 $\mathrm{mm}$ crack at $28.5^{\circ}$; crack at the trailing edge increases to $7-8 \mathrm{~mm}$ at $30^{\circ}$ and about $1 \mathrm{~mm}$ crack also appears at the leading edge; the crack at the trailing edge reaches $1 \mathrm{~cm}$ at $31^{\circ}$ and that at the leading edge increases to $5 \mathrm{~mm}$; the crack at trailing edge further extends to $2 \mathrm{~cm}$ at $31.5^{\circ}$ and the shear crack also appears at the lateral edge; about $1 \mathrm{~mm}$ and $2 \mathrm{~mm}$ circular cracks respectively appear at the area between $120 \mathrm{~cm}-130 \mathrm{~cm}$ of scale and around the area and leading edge crack is up to $7-8 \mathrm{~mm}$. Landslide deformation is remarkable at $31.5^{\circ}$. See Figure 4 for the landslide under the critical state.

See the displacement coordinate for pipeline at different angles. As shown in Figure 5, the central part of pipeline is featured with the maximum deformation, i.e. the part inside of landslide body is with the most evident deformation, and the maximum displacement of central part is $11 \mathrm{~mm}$, indicating its degree of damage is the highest.

The stress, calculated from strain computation and varying with pipeline length, is as shown in Figure 6.

Strain and stress, varying with pipeline length when the landslide body is under the critical state (i.e. $31.5^{\circ}$ ), are as shown in Figure 7. 
Assuming that the external force distribution is in the parabolic form, it can be expressed as:

$$
q=a x^{2}+b x+c
$$

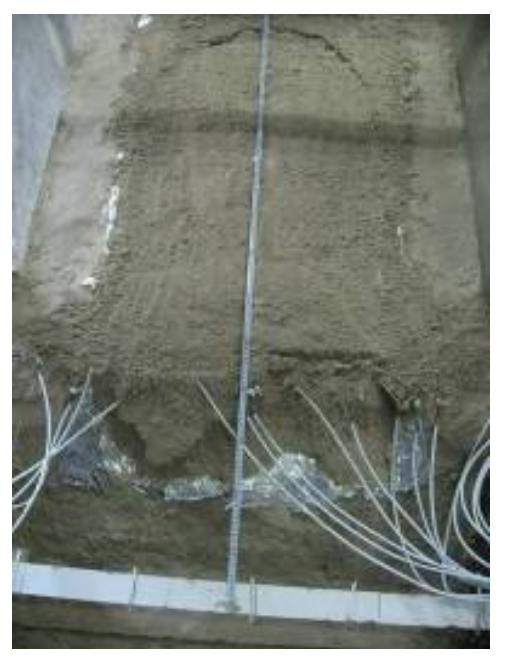

Figure 4. Critical state of landslide body with thickness of $10 \mathrm{~cm}$.

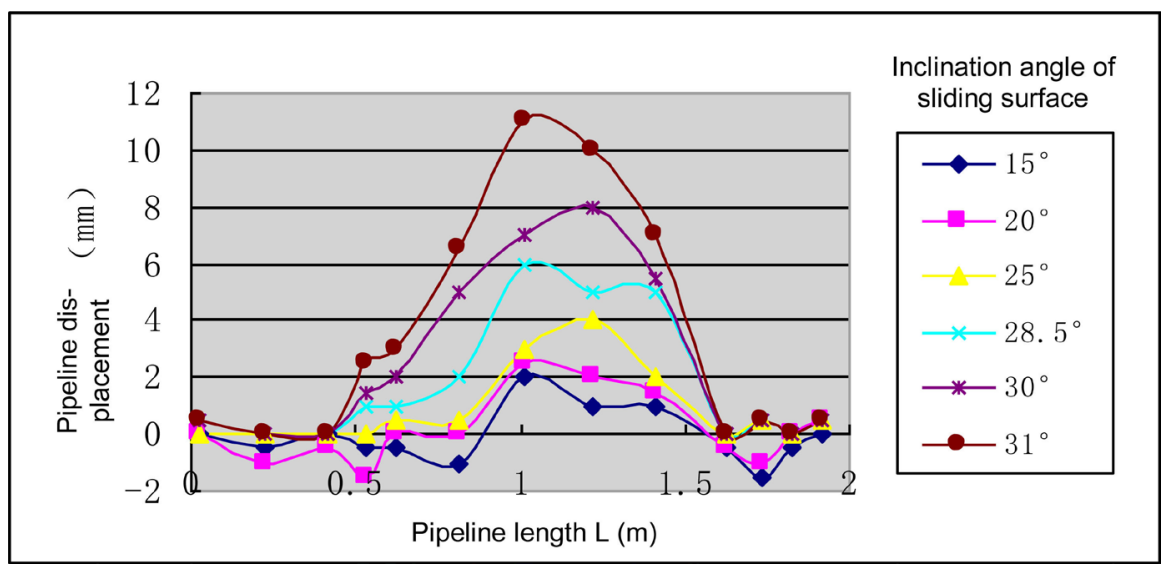

Figure 5. Diagram of pipeline displacement varying with coordinates.

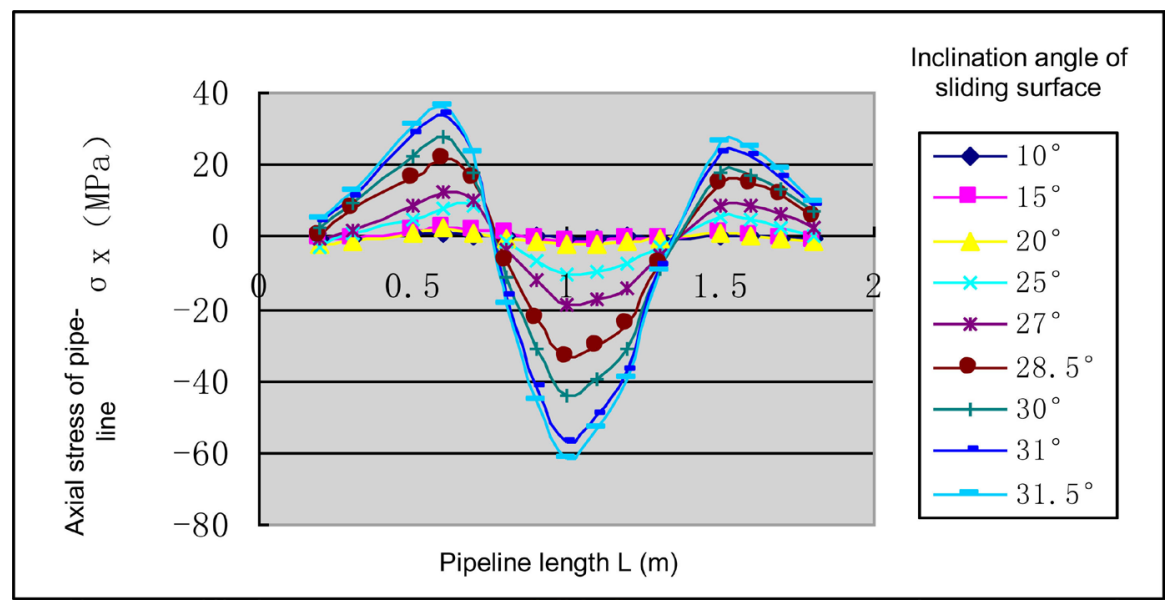

Figure 6. Diagram of positive axial stress varying with pipeline length. 


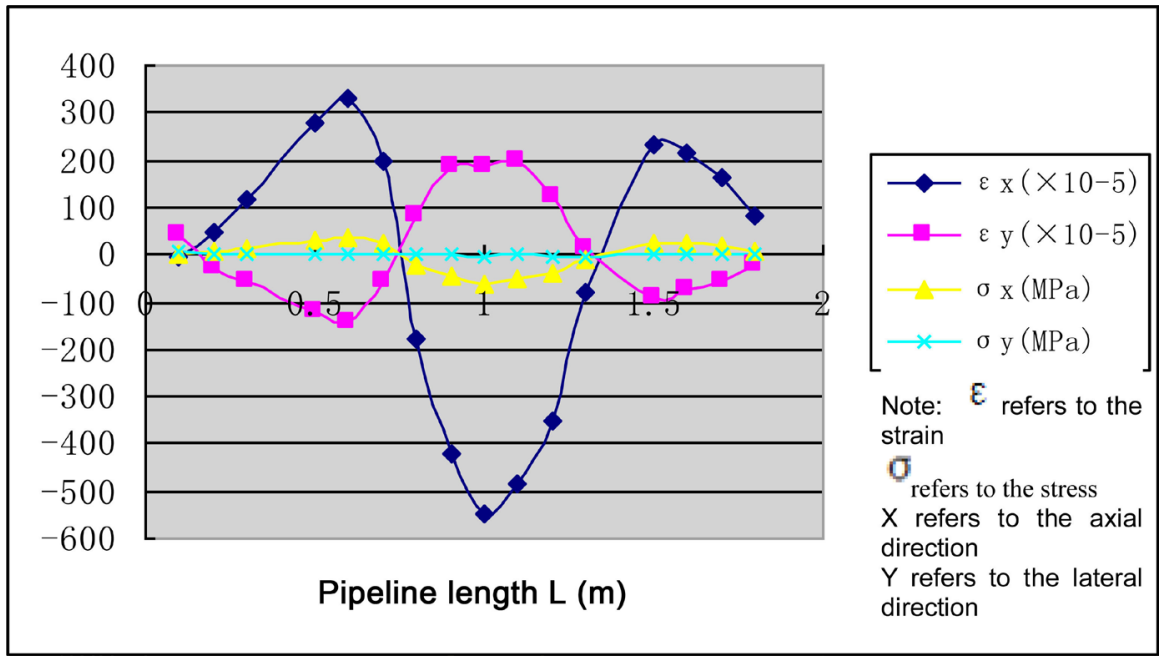

Figure 7. Strain and stress varying with pipeline length.

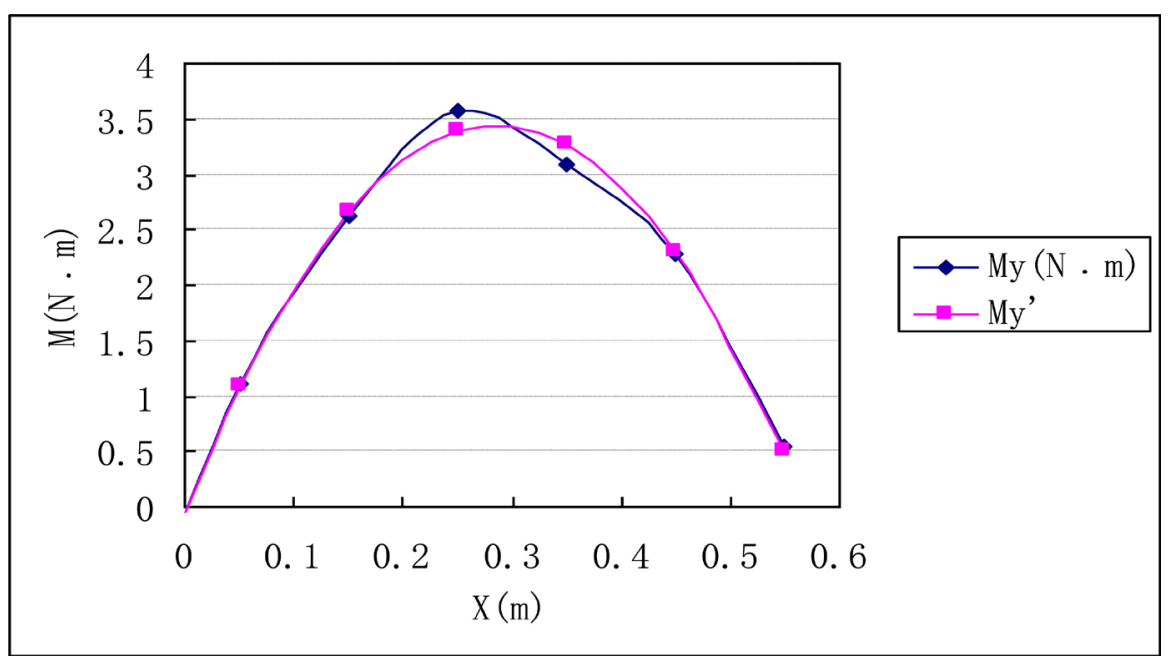

Figure 8. Calculated bending moment and trial bending moment varying with coordinates.

The bending moment of pipeline is:

$$
M=-\frac{1}{12} a x^{4}-\frac{1}{6} b x^{3}-\frac{1}{2} c x^{2}+\frac{1}{2}\left(\frac{a}{3} l^{3}+\frac{b}{2} l^{2}+c l\right) x
$$

The internal stress bending moment My, calculated from axial stress through inverse computation, is fitted into the bending-moment curve by Formula (2), to generate the bending moment My', as shown in Figure 8. Both are featured with favorable similarity with each other and their correlation coefficient is 0.998 . It indicates that assuming distribution of landslide force on pipeline as the parabolic form is reasonable.

The distribution of force $(q)$ applied by landslide to pipeline can be deduced and its equation is as follow:

$$
q=-x^{2}+0.572 x+84.1
$$

Analyze and process 6 groups of test data and adopt the linear fitting analysis 
method: Firstly, draw the scatter diagram and the regression line with measured (test) data, and identify the approximation relation between independent variable and dependent variable through the principle of least square method, then acquire the actual external force according to trial calculation and similarity principles. After that, calculate the actual stress on the basis of the actual external force. Finally, evaluate pipeline vulnerability in case of landslide on the basis of calculation results.

\subsection{Acting Force of Pipeline in Case of Landslide}

Acquiring the acting force $q$ of landslide body to oil and gas pipeline is the key to study safety issue of pipeline in case of landslide. The function relationship between the acting force $q$ and landslide indexes can be established through physical tests.

As indicated by test results, the external force from landslide on pipeline is a distribution function of pipeline, and its functional form may be assumed as $q=a x^{2}+b x+c \quad$ (unit: $\mathrm{N} / \mathrm{m}$ ). Since the symmetric constant $c$ is the external force distribution of that with coordinates of 0 and 1 , on which the external force depends, three parameters ( $a, b$ and $c$ ) in the forcing function may be analyzed. a determines the opening direction of parabolic load, and the negative a indicates that the opening direction is downward. In addition, $a$ and $b$ jointly determine the crook degree of parabolic load. Assuming $a=-1$ while $x=1$ and the external load is $c, a l+b=0$ and $b=1$ may be deduced. According to the above-mentioned analysis, it can be concluded that Coefficient $c$ is the key value determining the external load. The function relationship between the constant $c$ and landslide may be identified through regression and fitting analysis:

1) Calculation function varying with thickness $H_{h}$ of landslide area

$$
C_{h}=17.383\left(\frac{\alpha / \varphi}{e^{H_{h}}}\right)^{4.0342}
$$

2) Calculation function varying with width $B$ of landslide area

$$
C_{B}=15.194\left(\frac{\alpha / \varphi}{e^{0.15 B}}\right)^{3.0687}
$$

3) Calculation function varying with length $L$ of landslide area

$$
C_{L}=169.29\left(\frac{\alpha / \varphi}{e^{L}}\right)^{5.1405}
$$

4) Calculation function varying with pipeline buried depth $h_{m}$

$$
C_{h_{m}}=9.0586\left(\frac{\alpha / \varphi}{e^{h_{m}}}\right)^{4.142}
$$

As indicated by above-mentioned analysis, $c$ is featured with favorable correlation ( $R^{2}$ is greater than 0.73) when being fitted with the landslide index parameters $H_{h}, B, L$ and $h_{m}$ through $c=a(f(\alpha, \varphi, \xi))^{b} \quad$ (where, $a$ and $b$ are constants and $\xi=\left(H_{h}\right.$ or $B$ or $L$ or $\left.\left.h_{m}\right)\right)$. Therefore, all of them are comprehensively considered and the overall fitting expression (mean value), the upper boundary 
fitting expression (the maximum) and the lower boundary expression (the minimum) are given, as shown in Figure 9.

It shows a superior fitting relation, and relation equations between $c$ and landslide index parameters $H_{h}, B, L$ and $h_{m}$, and between inclination angle and internal friction angle of sliding surface. In addition, the upper boundary relation equation and the lower boundary relation equation are given. See Formula (10)-(31)-Formula (10)-(33) for details.

Overall fitting relation:

$$
c=0.3513 \times e^{3.2276 \times\left(\frac{\alpha / \varphi}{e^{0.02 \times\left(H_{h}+0.15 B+L+h_{m}\right)}}\right)}
$$

Upper boundary fitting relation (maximum):

$$
c_{\max }=16.477 \times\left(\frac{\alpha / \varphi}{e^{0.02 \times\left(H_{h}+0.15 B+L+h_{m}\right)}}\right)^{4.1253}
$$

Lower boundary fitting relation (minimum):

$$
c_{\min }=1.6425 \times\left(\frac{\alpha / \varphi}{e^{0.02 \times\left(H_{h}+0.15 B+L+h_{m}\right)}}\right)^{6.7194}
$$

where: $c$-Constant of external force equation in indoor physical model;

$\alpha, \varphi$-Inclination angle and internal friction angle $\left({ }^{\circ}\right)$ of sliding surface of landslide area in indoor physical model.

$H_{h}, B, L$ and $h_{m}$-Thickness, width and length of landslide body and buried depth of pipeline in indoor physical model (unit: $\mathrm{m}$ ).

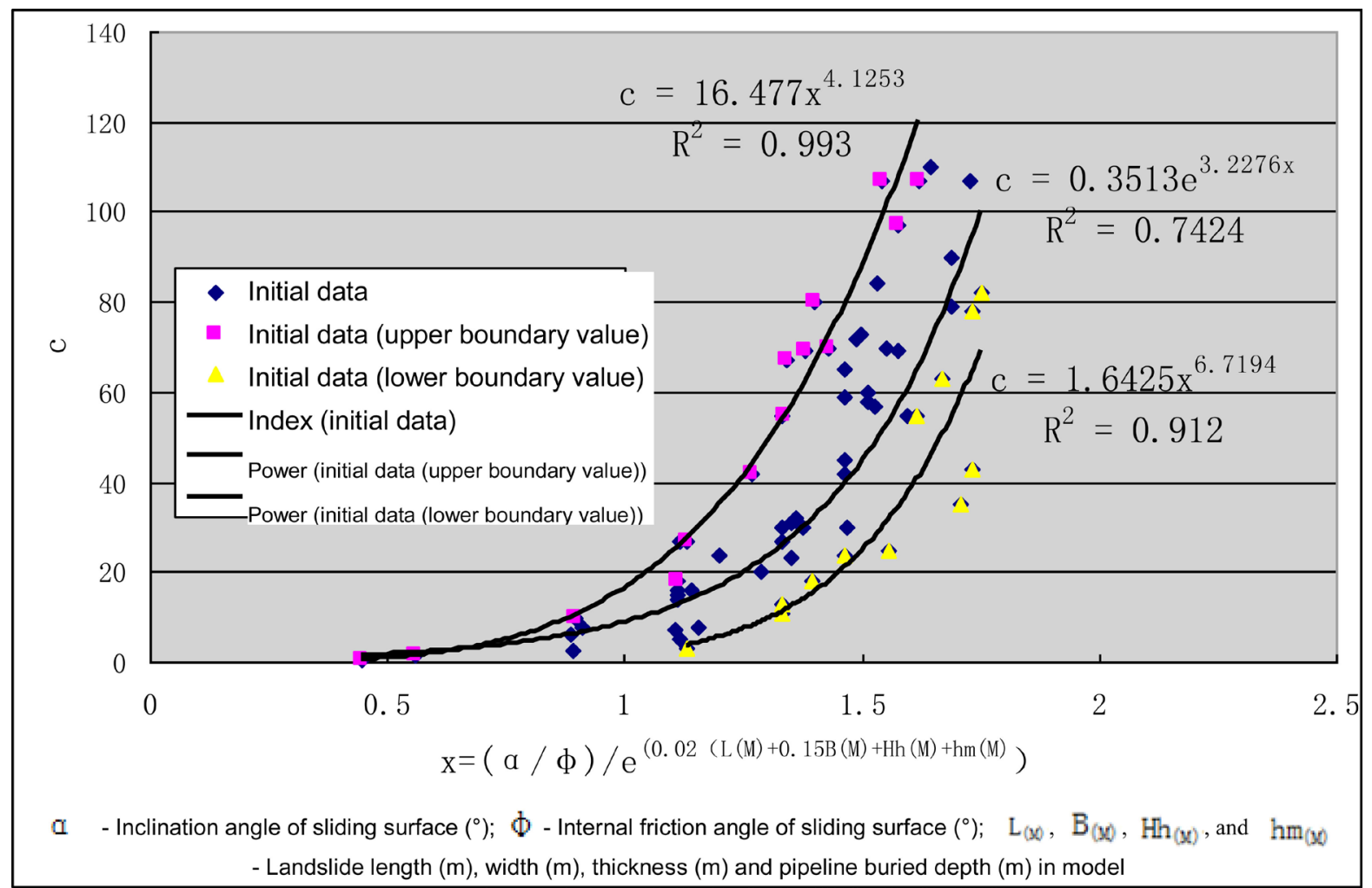

Figure 9. Curve chart of relation between $c$ and landslide index parameter. 


\section{Conclusion and Suggestion}

This project, taking pipeline transversely crossing landslide as an example and adopting physical simulation methods, studies vulnerability of oil and gas pipeline in case of landslide, discusses and preliminarily establishes the expression of landslide under different characteristic conditions to acting force of oil and gas pipeline. As indicated by the preliminary results, the acquired physical simulation results of oil and gas pipeline vulnerability is featured with favorable practicability in case of landslide, but those simple, applicable and practical empirical equations need to be further verified with many living examples and evaluation methods shall be modified on the basis of verification results accordingly.

\section{References}

[1] Savigny, K.W., Porter, M. and Leir, M. (2011) Geohazard Risk Management for the Onshore Pipeline Industry: Exploration \& Production. The Oil \& Gas Review, 2005. http://www.bgcengineering.com/en/geohazard-riskmanagement-onshore-pipeline-i ndustry

[2] OTH Bruschi, R., et al. Pipelines Subject to Slow Landslide. Movements Structural Modeling vs Field Measurement. Proceedings of 15 th International Conference on Offshore Mechanics and Arctic Engineering (ASME-OMAE 1996), Volume V, Pipeline Technology.

[3] Chen, L.Q., Song, L.Q., Wu, S.J., et al. (2017) FEM-Based Stress Analysis of Gas Pipelines in Landslide Areas. Natural Gas Industry, 37, 84-91.

[4] Liu, B., Wu, Z.Z., Liu, J.X. and Zhang, H. (2011) The Strain-Based Design of Buried Pipeline Subjected to Landslides. Computational Methods in Structural Dynamics and Earthquake Engineering, 25-28 May 2011, Corfu, Greece.

[5] Han, B., Wang, Z.Y., Zhao, H.L., Jing, H. and Wu, Z.-Z. (2012) Strain-Based Design for Buried Pipelines Subjected to Land-Slides. Petroleum Science, 9, 236-241. https://doi.org/10.1007/s12182-012-0204-y

[6] Hao, J.B., Liu, J.P., Jing, H.Y., et al. (2012) A Calculation of Landslide Thrust Force to Transverse Pipelines. Acta Petrochina Sinica, 33, 1093-1097.

[7] Lian, Z.F. and Li, F.L. (2014) Analysis of Mechanical Strength of Buried Pipelines in Landslide Areas. Journal of Southwest Petroleum University (Science \& Technology Edition), 36, 165-170. 